\title{
Suicidal ideation and psychological wellbeing of adolescents in Galle education division
}

\author{
Lokanwaththa $\mathrm{CS}^{1}$, Ponnamperuma TT ${ }^{2}$ \\ ${ }^{1}$ Department of Nursing, Faculty of Allied Health Sciences, University of Ruhuna, Galle, Sri Lanka. \\ ${ }^{2}$ Department of Community Medicine, Faculty of Medicine, University of Ruhuna, Galle, Sri Lanka.
}

\author{
Correspondence: $\quad$ Ms. C. S. Lokanwaththa \\ e-mail: c.s.lokanwaththa@gmail.com \\ (D) https://orcid.org/0000-0002-3466-269X \\ Submitted on 24.11.2020 and accepted for publication on 22.12.2020
}

\begin{abstract}
Introduction: Suicide is a global health problem affecting individuals of all ages. Sri Lanka shows a high suicide rate with a considerable number reporting from young age group. As suicidal ideation are present in victims before committing suicide. Therefore, this study was designed to assess suicidal ideation among adolescents in Galle, Sri Lanka. Besides, the study assessed the general psychological wellbeing and factors associated with it in youth.
\end{abstract}

Methods: School-based screening was conducted in a conveniently selected sample of students from six national schools in the Galle educational division. Questionnaires assessed lifetime suicidal ideation, attempts, and present psychological well-being (General Health Questionnaire $\left.\mathrm{GHQ}_{30}\right)$.

Results: There were 1479 participants, age 16-19 years with 52\% females. The lifetime prevalence of suicidal ideation in the sample was $22 \%$ with $11 \%$ having active ideation. Previous suicidal attempts were $4 \%$. Compared to participants with no suicidal ideation, individuals with passive and active ideations had poor psychological well-being $(\mathrm{F}=152.86, p<0.001)$. In logistic regression, the GHQ score predicted suicidal ideation $[\mathrm{OR}=1.17$, $p<0.001]$. Further, present psychological wellbeing was associated with age, gender, socioeconomic status, happiness at school, and happiness at home, explaining $18.6 \%$ variances in the regression model.

Conclusion and Recommendations: There is a considerably higher number of adolescents having suicidal ideation, and therefore, it is crucial to screen and identify these adolescents for help. Proper sustainable programs need to be implemented for this purpose and attention should be paid to improve the socioeconomic status, happiness at school, and happiness at home. Further research is recommended to obtain more in-depth information on suicidal ideations.

Keywords: Adolescent, General Health Questionnaire (GHQ), psychological wellbeing, suicide, suicidal idealtion

\section{Introduction}

Suicide occurs throughout the world, affecting individuals of all ages. Importantly, suicide has been reported as the second leading cause of death among the youth (1). In recent decades, Sri Lanka is recorded as a leading country with the highest rate of suicide. In 1995, the country ranked first in the world with a 47/100,000 suicidal rate (2). Thereafter the rate has gradually declined, and the latest statistics report a suicide rate of 15 - 19 persons per 100,000 populations (3). 
A report published by the National Institute of Mental Health in September 2011 states that a majority of the victims were in the age category 15 - 44 years (4). Therefore, similar to adults, adolescents are at risk of committing suicide.

Suicidal ideations are thoughts of wanting to end one's own life and they are experienced by the individual before committing suicide. There are two kinds of suicidal ideation: passive and active. Passive suicidal ideation implies an existing desire to die, without a specific plan to carry out death. Active suicidal ideation implies an existing desire to die accompanied by a plan for how to carry out death (5). Although not all suicidal ideations lead to attempted or completed suicide, these thoughts generally exist prior to committing suicide (6). From the clinician's point of view, the severity of suicidal risk runs along a continuum, ranging from suicidal ideation alone to suicidal ideation with a plan, and suicidal attempts (7). However, predicting an individual for risk of committing suicide based on thoughts and plans, is often difficult as the relationship between these is not always linear. Therefore, suicidal ideation cannot always predict the risk of committing suicide but yet they cannot be neglected either.

Suicidal ideations are associated with many different factors. The strongest risk factors reported are previous suicidal attempts, family history of suicidal behaviours, and having psychiatric illnesses such as depression and other psychotic conditions. These few factors are common for all ages $(8,9,10)$, but there are several other factors particularly noted among the young age group. They are problems at homes such as poor parent-child attachment, domestic violence exposure, stressful life events, and problems at school such as peer problem and bullying (11). Furthermore, there is a gender paradox in youth suicidal behaviour. Girls have shown a higher rate of suicidal ideation and suicidal attempts while boys showed a higher rate of committing suicide (12). However, in Sri Lanka, the suicide rate in young girls 10 - 19 years has been reported slightly higher than in boys (13).

There are only a few studies in the area of suicide was conducted in Sri Lanka, and to the best of the authors' knowledge no study was conducted to identify suicidal risk in the young age group. Further, suicide is a preventable cause of death, and risk adolescents can be easily identified by conducting a screening program at schools. Therefore, this study was designed as a schoolbased screening to identify adolescents with risk of suicide. We hope that the finding of this study will draw the attention of responsible authorities to take necessary preventive actions to minimise youth suicide. In order to accomplish this strategy, the present study investigated the following;

1) Lifetime suicidal ideation. We explored lifetime suicidal thoughts, plans, and attempts among adolescents. In addition, we inquired about possible reasons for suicidal ideation.

2) Present psychological wellbeing. We believed that the present psychological wellbeing associates with suicidal ideations, and therefore examined the predictability of the general psychological state to suicidal ideation.

3) Factors affecting psychological wellbeing. We believed improving present psychological wellbeing will help to prevent suicide, and therefore assessed the factors associated with present psychological wellbeing.

\section{Methods}

\section{Setting and participants}

The study was conducted in South of Sri Lanka, in the Galle education division. This area has nine national schools and twenty-one provincial schools. We selected national schools as they have Advanced Level classes of all four subject streams (Bioscience, Mathematics, Commerce, Arts) in the Sri Lankan education system. Among the national schools, we selected six schools purposefully to include three girl's schools and three boy's schools. From these schools, we selected two classes randomly from each subject stream (eight classes from each school), and recruited all children $(n=1479)$ who were present on day of data collection for the study.

Ethical approval was obtained from the Ethics Review Committee, Faculty of Allied Health Sciences, University of Ruhuna. Permission was taken from the Ministry of Education, principals and class teachers. Written informed consent was obtained from the parents and participants. Data collection was done at a convenient time for the students without disturbing their school activities. 


\section{Measures}

A self-administered Questionnaire was used to collect data. It comprised of three sections;

Section one: This section assessed demographic information, happiness at school, happiness at home, and socioeconomic status. The happiness at home and school were assessed by two questions, each measured in 5 point likert scale ranging from $5=$ very happy to $1=$ very unhappy. Socioeconomic status was measured by a Subjective Social Status (SSS) Scale ranged from 0 - 9(14).

Section two: This section assessed the suicidal ideation. Questions assessed the lifetime suicidal thoughts, suicidal plans, and previous suicidal attempts. Isolated suicidal thoughts were considered as having 'passive suicidal ideation', and having thoughts with suicidal plans or attempts were considered as having 'active suicidal ideation'. Further, they were asked to report possible reasons for their suicidal thoughts.

Section three: This section assessed the current psychological wellbeing by the General Health Questionnaire-30 $\left(\mathrm{GHQ}_{30}\right)$. It is a validated questionnaire for the country and it is widely used in the primary care setting (15). This questionnaire has 30 questions (range $0-30$ ) and higher scores indicated poor psychological wellbeing. Samaraweera et al., (2010) study this questionnaire with the Beck's scale for suicidal ideation and proved that GHQ categories were compatible identifying suicidal ideation in Sri Lanka population (16).

\section{Statistical analysis}

Analyses were performed with SPSS, with two-tailed $p \leq 0.05$ considered significant. Descriptive statistics were used to describe the main demographic information, and other variables in the study. ANOVA was conducted to compare total GHQ scores between active, passive and no suicidal ideation, and also between four advanced level school subject streams. Logistic regression was conducted to see the predictive ability of the GHQ score on suicidal ideation. Pearson correlation was conducted to observe inter-correlations between GHQ total score, socioeconomic status, happiness at home and happiness at school of the adolescents.
To identify the associated factors for present psychological wellbeing, linear regression analysis was conducted taking $\mathrm{GHQ}_{30}$ total score as the dependent variable, and demographic variables, socioeconomic status, happiness at home and happiness at school as independent variables. Those variables with significant effect in the preliminary models were retained in the final models.

\section{Results}

\section{Sample characteristics}

There were 1479 students, age range $16-19$ years (mean $=17.69, \mathrm{SD}=0.48)$ participated for the study. Most of the students scored average in the SSS scale representing the middle social class (mean $=5.38, \mathrm{SD}=1.72$ ). All the other demographic information are described in Table 1. Students reported that they were happy at home $($ mean $=4.32$ $\mathrm{SD}=0.95$, range $=0-5$ ), and happy at school (mean $=3.95, \mathrm{SD}=1.34$, range $=0-5$ ). Several students reported that they were previously treated by a psychologist / psychiatrist for some psychological problem $(\mathrm{n}=70,4.8 \%)$. Among the participants, $11.7 \%$ (boys $n=168$ and girls $n=9$ ) have used alcohol, cigarettes or other substances.

\section{Suicidal ideation}

The lifetime prevalence of suicidal ideation in the total sample was $21.9 \%(\mathrm{n}=324)$. Among them $11.3 \%(\mathrm{n}=167)$ had passive suicidal ideation and $10.6 \%(\mathrm{n}=157)$ had active suicidal ideation. Previous suicidal attempts were reported by 55 (3.7\%) of adolescents. There was no observed gender difference in life time prevalence of suicidal ideation $\left.\chi^{2}(1,1479)=0.42, p=0.84\right)$ or in attempted suicide $\left.\chi^{2}(1,1479)=0.84, p=0.36\right)$. Socioeconomic status was significantly low in adolescents who had suicidal ideation (active or passive) compared to the others without suicidal ideation $(t(1414)=$ $-2.085, p=0.04)$. Adolescents having no-suicidal ideation were much happier at school and at home, compared to adolescents having suicidal ideation $(t(1464)=-5.79, p<0.001, t(1466)=-8.53$, $p<0.001$ respectively). Further, we asked their possible reasons for having suicidal ideations, and frequently reported reasons were stress due to studies $(30 \%)$, family conflicts $(27 \%)$, relationship 
problems ( $16 \%)$, problems with peers $(3 \%)$, bullying (3\%) and problems with siblings (2\%).

\section{Present psychological wellbeing}

We analysed the GHQ after excluding the participants who reported previous psychological problems $(\mathrm{n}=1409)$. As expected, adolescents showed a low GHQ mean score $=3.88(S D=5.27$, range 0 - 28) indicating good general psychological wellbeing. Girls reported a significantly higher score than boys 4.62 vs 3.06 respectively ( $t$ (1400) $=5.65, p<0.001)$. GHQ score significantly differed between Advanced Level subject streams $(F(3,1376)=11.59, p<0.001)$.
The post-hoc comparison revealed students learning arts subjects (mean $=2.72$ ) had a significantly lower GHQ score compared to students learning mathematics $($ mean $=4.75)$, biology $($ mean $=4.40)$ and commerce $($ mean $=4.24)$. The difference between the other subjects were not statistically significant (all $p>0.05)$.

We compared the GHQ scores between participants who had no suicidal ideation, passive suicidal ideation (suicidal thoughts only) and active suicidal ideation (suicidal thoughts with plans and or attempts).

Table 1: Demographic characteristics

\begin{tabular}{|c|c|c|}
\hline Demographic characteristic & $\mathbf{N}$ & $\%$ \\
\hline \multirow{2}{*}{ 1. Gender $-\begin{array}{l}\text { Female } \\
\text { Male }\end{array}$} & 761 & $51.5 \%$ \\
\hline & 712 & $48.5 \%$ \\
\hline \multirow{4}{*}{$\begin{aligned} & \text { 2. Religion }- \text { Buddhist } \\
& \text { Muslim } \\
& \text { Christian } \\
& \text { Hindu }\end{aligned}$} & 1322 & $89.4 \%$ \\
\hline & 41 & $2.8 \%$ \\
\hline & 14 & $0.9 \%$ \\
\hline & 2 & $0.1 \%$ \\
\hline \multirow{4}{*}{$\begin{array}{r}\text { 3. Advanced Level subject stream - } \text { Biology } \\
\text { Mathematics } \\
\text { Commerce } \\
\text { Arts }\end{array}$} & 356 & $24.1 \%$ \\
\hline & 352 & $23.8 \%$ \\
\hline & 304 & $20.6 \%$ \\
\hline & 441 & $29.8 \%$ \\
\hline \multirow{4}{*}{$\begin{aligned} \text { 4. Living place }- & \text { Home with parents } \\
& \text { Relative's home } \\
& \text { Boarding place } \\
& \text { School hostel }\end{aligned}$} & 1381 & $89.4 \%$ \\
\hline & 97 & $2.5 \%$ \\
\hline & 115 & $7.8 \%$ \\
\hline & 3 & $0.2 \%$ \\
\hline
\end{tabular}

Table 2: GHQ Measures: Descriptive statistics and ANOVA results for groups defined according to suicidal ideation Suicidal Ideation GHQ Score

\begin{tabular}{lrrrrr}
\hline \multirow{2}{*}{ Suicidal Ideation } & \multicolumn{5}{c}{ GHQ Score } \\
\cline { 2 - 6 } & $\mathbf{N}$ & Mean & SD & F & Post-hoc \\
\hline No suicidal ideation & 1070 & 2.82 & 4.01 & 152.86 & $\mathrm{a}<\mathrm{b}, \mathrm{c}^{* * *}$ \\
Passive suicidal ideation & 167 & 5.07 & 5.68 & & $\mathrm{~b}<\mathrm{c}^{* * *}$ \\
Active suicidal ideation & 157 & 9.82 & 7.59 & \\
\hline
\end{tabular}

Notes. GHQ $=$ General health questionnaire. $\mathrm{a}=$ no suicidal ideation, $\mathrm{b}=$ passive suicidal ideation, $\mathrm{c}=$ active suicidal ideation. $* * * p<0.001$ 
We compared the GHQ scores between participants who had no suicidal ideation, passive suicidal ideation (suicidal thoughts only) and active suicidal ideation (suicidal thoughts with plans and or attempts).

As shown in Table 2, the GHQ score significantly differed between the three groups. Post-hoc comparisons revealed the lowest GHQ total score among participants with no suicidal ideation compared to the other two groups, and the participants with passive suicidal ideation scored significantly lower than the adolescents with active ideation.

Next, we examined whether the GHQ score could predict suicidal ideation in adolescents. Logistic regression was conducted taking suicidal ideation (dummy-coded having suicidal ideation $=1$ ) as the dependent variable, and the GHQ score, age, and gender (dummy-coded female $=1$ ) as independent variables. The GHQ score significantly predicted suicidal ideation. Gender also showed a significant effect but not the age. The final model is summarised in the Table 3 .

As shown in Table 4, the GHQ total score was significantly and negatively correlated with age, socioeconomic status, happiness at home and happiness at school. According to the linear regression analysis results, GHQ score was significantly predicted by age, gender (dummycoded female $=1$ ), socioeconomic status, happiness at home and happiness at school (Table 5). In the final model, all five significant predictors together explained $18.6 \%$ of the variance in GHQ total scores $(F(5,1269)=58.02, p<0.001$.

Table 3: Logistic regression estimates for prediction of suicidal thoughts by GHQ scores

\begin{tabular}{lcccccc}
\hline Independent Variable & $\mathbf{B}$ & $\mathbf{S E}$ & $\mathbf{Z}$ & $\boldsymbol{p}$ & OR & CI \\
\hline GHQ & 0.156 & 0.013 & 139.226 & $<0.001$ & 1.169 & $1.139-1.200$ \\
Gender & -0.378 & 0.148 & 6.553 & 0.010 & 0.685 & $0.513-0.915$ \\
Age & -0.095 & 0.147 & 0.414 & 0.520 & 0.910 & $0.681-1.214$ \\
Socioeconomic status & 0.004 & 0.041 & 0.008 & 0.928 & 1.004 & $0.926-1.088$ \\
\hline
\end{tabular}

Notes: The dependent variable is suicidal ideation (coded 1 present, 0 absent). Gender (coded 0 male, 1 female). GHQ $=$ General health questionnaireUnderstanding that the psychological wellbeing measured by GHQ could predict suicidal ideation, we intended to identify what factors affect adolescent's current psychological wellbeing.

Table 4: Descriptive statistics and inter correlation

\begin{tabular}{lrrrrrrr}
\hline Variable & \multicolumn{7}{c}{ Pearson correlation } \\
\cline { 2 - 8 } & $\mathbf{N}$ & Mean & SD & $\mathbf{1}$ & $\mathbf{2}$ & $\mathbf{3}$ & $\mathbf{4}$ \\
\hline GHQ total & 1387 & 3.87 & 5.26 & & & & \\
Age & 1338 & 17.69 & 0.48 & $-0.65^{*}$ & & & \\
Socio-economic status & 1328 & 5.38 & 1.72 & $-0.11^{* *}$ & -0.04 & & \\
Happiness at home & 1379 & 4.32 & 0.95 & $-0.34^{* *}$ & -0.01 & $0.09^{* *}$ & \\
Happiness at school & 1374 & 3.92 & 1.06 & $-0.24^{* *}$ & -0.01 & 0.04 & $0.30^{* *}$ \\
\hline
\end{tabular}

Notes. GHQ $=$ General health questionnaire. ${ }^{*} p<0.05,{ }^{* *} p<0.01$ 
Table 5: Multiple regression estimates for variables associated with GHQ scores

\begin{tabular}{lcccc}
\hline Independent variable & B & SEB & $\beta$ & \multicolumn{1}{c}{$\boldsymbol{p}$} \\
\hline Age & -0.751 & 0.277 & -0.069 & 0.007 \\
Gender & 2.084 & 0.271 & 0.197 & $<0.001$ \\
Socio-economic status & -0.310 & 0.078 & -0.102 & $<0.001$ \\
Happiness at home & -1.598 & 0.147 & -0.289 & $<0.001$ \\
Happiness at school & -0.819 & 0.131 & -0.166 & $<0.001$ \\
\hline Notes. The dependent variable is GHQ (General health questionnaire score). Gender is coded 0 male, 1 female.
\end{tabular}

\section{General Discussion}

The current study assessed the previous suicidal ideation and current psychological well-being of advanced level school students in the Galle educational division. This study conducted with the participation of only national schools because of the requirement of representation from all four advanced level subject streams in the study sample.

Our first goal was to assess the suicidal ideation of adolescents. We asked questions regarding lifetime experience of suicidal thoughts, plans, and previous suicidal attempts. The lifetime prevalence of suicidal ideation (active and passive) among adolescents was high as $22 \%$, and a half $(11 \%)$ of them had active suicidal ideation (having suicidal thoughts with plans to commit suicide). It was alarming to identify that $55(4 \%)$ adolescents had previous suicidal attempts. Our findings are not different from the global school-based health survey done in the country in the year 2016, where they identified $6.5 \%$ (CI 4.8 - 8.6) of students' age 13 - 16 years had attempted suicide within one year of the survey (17). We observed no gender differences in suicidal ideation or in attempted suicide even though the study conducted from 1995 - 2011 showed higher rate of suicide in girls in the country (13). In line with previous studies low family income was related to more suicidal ideation in adolescents (16).

We further inquired about individuals' reasoning for suicidal ideation. Adolescents have reported issues related to three broad categories: i) Problems at school: stress due to studies, peer group rejections and bullying at the school ii) Problems at home: family conflicts and problems with siblings. iii) Problems with relationships: breakup of love affairs. Our findings are supported by many previous studies that identified similar problems. Lack of peer support and bullying were identified as reasons for suicidal ideation among Thai adolescents (11). A community survey conducted among Sri Lankans, age $15-64$ years, showed that family conflict is responsible for suicidal ideation among $60 \%$ of the participants (16). Relationship problems of adolescents were also previously reported for example sexual abuse, rejection, and teen dating violence $(9,18)$.

Our second goal was to identify the present psychological state of adolescents by $\mathrm{GHQ}_{30}$ and see whether the GHQ score could predict suicidal ideation. As expected GHQ mean score was lowest among adolescents with no suicidal ideation and highest among adolescents with active suicidal ideation. Analysis showed that GHQ was able to predict suicidal ideation. A population-based study in Sri Lanka also showed that GHQ could identify people (age 15 - 64 years) with suicidal ideation (16). Further, a study in Sudan also identified higher GHQ scores among women with suicidal ideation (19). GHQ may, therefore, be a good enough tool to identify adolescents at risk for suicidal ideation. Moreover, GHQ is a widely used, validated, short general checklist that has the practical advantage of easily understandable, easily administrable, and less time-consuming in collecting data in a large sample. Importantly, this questionnaire can be used for the purpose without intuition of thoughts. 
However, further studies are needed to recommend GHQ as a school-based screening tool for suicidal ideation.

Our last goal was to investigate factors that affect the current psychological wellbeing of adolescents. In line with our expectations, happiness at their school and home had significant effects. These two factors are also the two central problems that adolescents identified as reasons for having suicidal ideation. Addressing problems at school and at home will therefore improve present psychological wellbeing, and thereby possibly reducing suicidal ideation in adolescents. Schoolbased programs have been identified as a feasible and effective method of screening for mental health problems and suicide prevention. Screening of teenagers in schools in the United States has proved to be very effective in identifying the subgroup having suicidal thoughts and intervening towards their improvement (20).

The main strength of this study is the large sample, with almost full participation of adolescents in selected schools. This increases the reliability and generalisability of the results. The limitations we identified were, unavailability of a validated tool to measure suicidal ideation, measuring happiness at school and happiness at home using two single questions and the narrow age range of the adolescents.

\section{Conclusion and Recommendations}

A high percentage of suicidal ideation and a considerable number of adolescents with previous suicidal attempts were identified by this schoolbased screening. Study problems related to school and family were the main reasons adolescents identified as reasons for their suicidal ideation and poor general psychological status. Suicide is, therefore, a serious public health issue that needs timely interventions by specifically improving their situation at homes and at schools. Further, we suggest that the currently functioning school health inspection programs conducted by the Ministry of Health Sri Lanka can be easily improved to address this matter.

\section{Acknowledgements}

As authors we would like to give our gratitude to Senior Professor Chrishantha Abeysena for providing us the validated $\mathrm{GHQ}_{30}$ questionnaire for the study. Also we are thankful to the Zonal Educational Director, Principals and Teachers of the schools for permitting us to conduct the study. Especially, we are thankful for all participants and their guardians for their contribution towards the study.

\section{References}

1. WHO. Suicide prevention [English]. 2018 [Available from: https://www.who.int/health-topics/suicide\#tab=tab_1

2. Knipe DW, Metcalfe C, Fernando R, Pearson M, Konradsen F, Eddleston M, et al. Suicide in Sri Lanka 1975 - 2012: age, period and cohort analysis of police and hospital data. BMC Public Health. 2014; 14(1): 839.

3. de Silva VA, Senanayake SM, Dias P, Hanwella R. From pesticides to medicinal drugs: time series analyses of methods of self-harm in Sri Lanka. Bulletin of the World Health Organization. 2012;90(1): 40-46.

4. Amaranath S. Retrieved from World Socialist: https://www.wsws.org/en/articles/2012/09/sril-s28.html (Accessed on 22.01.2021).

5. Barry LC, Wakefield DB, Trestman RL, Conwell Y. Active and Passive Suicidal Ideation in Older Prisoners. Crisis. 2016;37(2): 88-94.

6. Senatore LR. Suicide Shouldn't Be a Secret. An Assessment of Adolescent Coping Strategies following a Suicide Prevention Program 2006. Available from: http://hdl.handle.net/10539/1566. (Accessed on 03.12.2018).

7. Greenhill LL, Waslick B. Management of suicidal behaviour in children and adolescents. The Psychiatric Clinics of North America. 1997; 20(3): 641-666.

8. Brent DA, Perper JA, Moritz G, Allman C, Friend A, Roth $\mathrm{C}$, et al. Psychiatric risk factors for adolescent suicide: a case-control study. J Am Acad Child Adolesc Psychiatry. 1993; 32(3): 521-529.

9. Fergusson DM, Woodward LJ, Horwood LJ. Risk factors and life processes associated with the onset of suicidal behaviour during adolescence and early adulthood. Psychol Med. 2000; 30(1): 23-39. 
10. Strandheim A, Bjerkeset O, Gunnell D, Bjørnelv S, Holmen TL, Bentzen N. Risk factors for suicidal thoughts in adolescence - a prospective cohort study: the Young-HUNT study. 2014; 4(8): e005867.

11. Peltzer K, Pengpid S. Suicidal ideation and associated factors among school-going adolescents in Thailand. Int JEnviron Res Public Health. 2012; 9(2): 462-473.

12. Cash SJ, Bridge JA. Epidemiology of youth suicide and suicidal behaviour. Curr Opin Pediatr. 2009; 21(5): $613-$ 619.

13. de Silva V, Hanwella R, Senanayake M. Age and sex specific suicide rates in Sri Lanka from 1995-2011. Sri Lanka Journal of Psychiatry. 2013;3(2): 7-11.

14. Goodman E, Adler NE, Kawachi I, Frazier AL, Huang B, Colditz GA. Adolescents' perceptions of social status: development and evaluation of a new indicator. Paediatrics. 2001;108(2): e31.

15. Abeysena C, Peiris U, Jayawardana P, Rodrigo A. Validation of the Sinhala version of 30-item General Health Questionnaire. Journal of the Postgraduate Institute of Medicine. 2014; 1
16. Samaraweera S, Sumathipala A, Siribaddana S, Sivayogan S, Bhugra D. Prevalence of suicidal ideation in Sri Lanka. Crisis. 2010; 31(1): 30-35.

17. CDC. Global School-based Student Health Survey (GSHS) 2016, 17.9.2018 [cited 2018 17]: [5 p.]. Available from: https://www.cdc.gov/gshs/countries/seasian/srilanka.htm

18. Kirkcaldy BD, Siefen GR, Urkin J, Merrick J. Risk factors for suicidal behaviour in adolescents. Minerva Pediatr. 2006; 58(5): 443-450.

19. Goldney RD, Harris LC, Badri A, Michael S, Fisher L. Suicidal ideation in Sudanese women. Crisis. 1998; 19(4): 154-158.

20. Friedman RA. Uncovering an epidemic--screening for mental illness in teens. $N$ Engl $J$ Med. 2006; 355(26): 2717-2719. 